\title{
Co-binding by YY1 identifies the transcriptionally active, highly conserved set of CTCF-bound regions in primate genomes
}

\author{
Petra C Schwalie ${ }^{1,5 \dagger}$, Michelle C Ward ${ }^{2,3+}$, Carolyn E Cain ${ }^{3}$, Andre J Faure ${ }^{1}$, Yoav Gilad ${ }^{3}$, Duncan T Odom ${ }^{2,4^{*}}$ \\ and Paul Flicek ${ }^{1,4^{*}}$
}

\begin{abstract}
Background: The genomic binding of CTCF is highly conserved across mammals, but the mechanisms that underlie its stability are poorly understood. One transcription factor known to functionally interact with CTCF in the context of X-chromosome inactivation is the ubiquitously expressed YY1. Because combinatorial transcription factor binding can contribute to the evolutionary stabilization of regulatory regions, we tested whether YY1 and CTCF co-binding could in part account for conservation of CTCF binding.

Results: Combined analysis of CTCF and YY1 binding in lymphoblastoid cell lines from seven primates, as well as in mouse and human livers, reveals extensive genome-wide co-localization specifically at evolutionarily stable CTCF-bound regions. CTCF-YY1 co-bound regions resemble regions bound by YY1 alone, as they enrich for active histone marks, RNA polymerase II and transcription factor binding. Although these highly conserved, transcriptionally active CTCF-YY1 co-bound regions are often promoter-proximal, gene-distal regions show similar molecular features.
\end{abstract}

Conclusions: Our results reveal that these two ubiquitously expressed, multi-functional zinc-finger proteins collaborate in functionally active regions to stabilize one another's genome-wide binding across primate evolution.

\section{Background}

CTCF is a highly conserved, 11-zinc finger multi-functional protein [1,2] important in regulating gene expression [3-5], insulating against enhancer-promoter interactions [6,7], regulating splicing [8], as well as ensuring allele-specific expression at imprinted genes [7] and on the inactive $\mathrm{X}$ chromosome [9]. Genome-wide studies have suggested that CTCF binding demarcates active and repressive domains [10-12] and contributes to nucleosome positioning [13], as well as nuclear organization and higher order chromatin structure [14].

CTCF's binding profile is largely (but not entirely [15]) invariant across mouse tissues [16], human cell lines [10] and divergent species compared to those of tissuespecific transcription factors (TFs) [17-22]. Comparisons

\footnotetext{
* Correspondence: duncan.odom@cruk.cam.ac.uk; flicek@ebi.ac.uk

${ }^{\dagger}$ Equal contributors

'European Molecular Biology Laboratory, European Bioinformatics Institute, Wellcome Trust Genome Campus, Hinxton, Cambridge CB10 1SD, UK

${ }^{2}$ University of Cambridge, Cancer Research UK-Cambridge Institute, Robinson Way, Cambridge CB2 ORE, UK

Full list of author information is available at the end of the article
}

of CTCF binding have revealed a high level of conservation in liver tissue of species separated by up to 180 million years [21], as well as in cell lines from human, mouse, and chicken [19]. Additionally, CTCF has been shown to bind transposable elements in both embryonic stem cells [18] and differentiated tissue [21]. While certain repeat elements have expanded CTCF target sites in several mammalian lineages, thus far there is no evidence of this process being prevalent in primates based on experiments in human and rhesus macaque [21].

The availability of sequenced primate genomes [23-26] and the ability to transform blood B cells into immortal lymphoblastoid cell lines (LCLs) with the Epstein-Barr virus (EBV) [27] facilitates functional genomics comparisons across different primate species. To date, such interprimate studies have been carried out primarily at the level of gene expression [28-32]. However, it had already been proposed in the 1970s that phenotypic differences between primates are largely due to regulatory differences [33]. While comparative evolutionary studies in mammals 
have provided insight into regulatory mechanisms, limited information is available within the primate order.

Inter-primate comparisons of regulatory evolution have been performed for histone modifications, which can explain $7 \%$ of gene expression differences among human, chimpanzee, and rhesus macaque cell lines [34]. Further, DNA methylation studies revealed that promoter methylation differences underlie 12 to $18 \%$ of gene expression differences between humans and chimpanzees and that approximately $10 \%$ of CpG islands are significantly differentially methylated between the two species $[35,36]$. Differences in the binding of transcriptional regulators have been inferred from the presence of several hundred species-specific DNase I hypersensitive sites near genes differentially expressed between humans and chimpanzees [37]. Regulatory DNA element comparisons among primates are emerging [38,39]; however, a comprehensive analysis of the binding of a sequence-specific factor such as CTCF across primate species has yet to be performed.

CTCF can exert its different functions through interactions with diverse protein factors [40,41]. One such factor is Yin Yang 1 (YY1), which was originally shown to trans-activate the Tsix ncRNA during X-chromosome inactivation through its interaction with CTCF [9]. There is a strong pattern of co-localization between these two factors at predicted boundary elements, suggesting that they could act synergistically in delimiting chromatin domains [42]. Genome-wide chromatin immunoprecipitation followed by high-throughput sequencing (ChIPseq) data have recently indicated global co-localization of CTCF and YY1 in human cells [43] with a specific distance constraint [44].

YY1 was first identified as both a repressor and an activator of the adeno-associated virus under different conditions [45], but, similar to CTCF, it has been attributed a broad range of distinct functions, including roles in imprinting [46-48], X-chromosome inactivation [49], and chromatin structure maintenance [50]. YY1 is essential in mouse development, as its deletion results in periimplantation lethality [51]. A homolog of YY1, the Drosophila PHO protein, is involved in Polycomb repression [52,53], but there is limited evidence for this in mammals, where YY1 is rather viewed as a global regulator. YY1 binding motifs are overrepresented in core promoters [54], with approximately $10 \%$ of human promoters containing it [55]. Additionally, YY1 is important for initiating transcription of various transposable elements such as LINE-1s [56,57], Alu SINEs [58], Herv-Ks [59] and LTRs [60].

Here, we map genome-wide CTCF binding at high resolution in seven primate species and propose that the evolutionary stability of CTCF genomic occupancy is, at least in part, linked to its co-binding with the ubiquitous TF YY1.

\section{Results}

\section{Evolution of CTCF binding in seven primates}

CTCF binding in distantly related mammalian species is highly conserved compared to that of tissue-specific TFs [17-22]. Here, we analyzed the evolution of CTCF binding at high resolution in LCLs from seven primates, spanning 40 million years of evolution, to determine what mechanism(s) contribute to binding conservation. We experimentally profiled CTCF in most of the great apes (Homo sapiens - H.sap, Pan troglodytes - P.tro, Gorilla gorilla - G.gor, and Pongo pygmaeus - P.pyg), two species of Old World monkey (Macaca mulatta - M.mul and Papio hamadryas - P.ham), and one species of New World monkey (Sanguinus oedipus - S.oed) (Table S1 in Additional file 1). In each species, we performed ChIP-seq in at least two replicates and used naked DNA (input) as control (Figure 1A; Additional file 1: Table S2). Species were aligned to their respective genome except for P.ham, which was aligned to the M.mul genome (84\% reads aligned), and S.oed, which was aligned to the Callithrix jacchus (C.jac) genome (65\% reads aligned), as there are currently no published genomes available for these species, and M.mul and C.jac represent the closest sequenced relatives. We determined regions of significant ChIP enrichment (referred to as 'bound regions' or 'binding events') compared to input samples with CCAT 3.0 (Materials and methods) using a fixed false discovery rate (FDR) of 0.05 across species (Materials and methods). Inter-species comparisons were made using Ensembl release 60 genome-wide 6-way EPO primate multiple alignments [61], which include H.sap, P.tro, G.gor, P.pyg, M.mul and C.jac (Table S1 in Additional file 1). Our universal cutoff approach is unbiased in that it does not assume that binding events are conserved across species; however, it favors a model where differences are more likely than shared binding and as such is likely to marginally underestimate the fraction of conserved binding events [21,22] (Materials and methods).

In each of the analyzed primates, we detected thousands of shared and species-specific CTCF-bound locations (Figures $1 \mathrm{~B}$ and $2 \mathrm{~A}$ ). To determine the extent of binding conservation, we split CTCF-bound regions into six different classes: (i) species-specific and not included in the genome-wide multiple alignments, (ii) species-specific and included in the alignments, (iii) shared with exactly one other species, (iv) shared with two, three, or four other species, (v) shared with exactly five other species (that is, missing in exactly one species), and (iv) shared across all primates (Figure 2A; Figure S2A in Additional file 1). On average, approximately $40 \%$ of CTCF-bound regions are shared across six or seven species (highly shared). Conversely, thousands of regions (on average $20 \%$ of all CTCF binding events) are species-specific or only shared with a single other species. The vast majority $(>80 \%)$ of CTCF- 


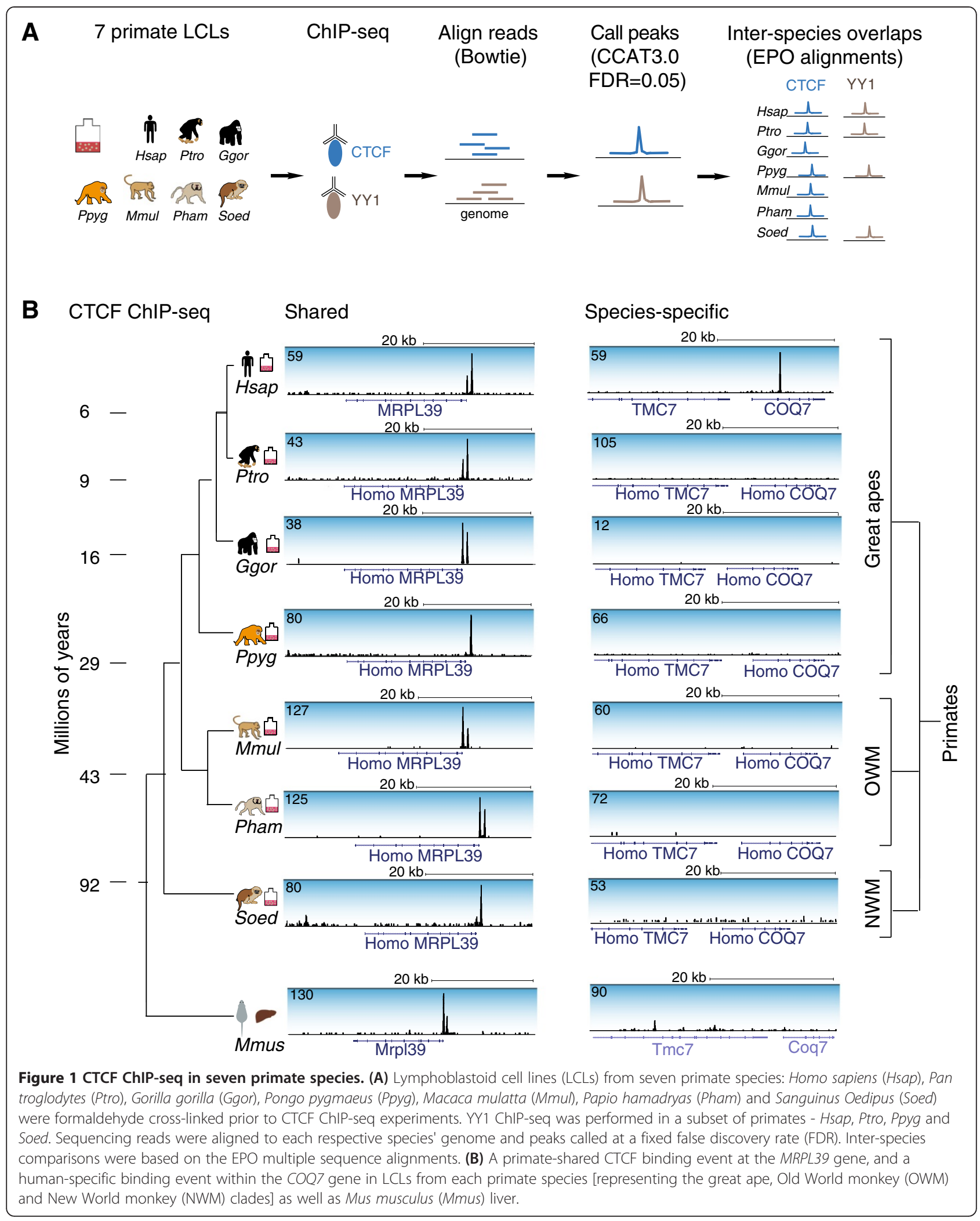


A

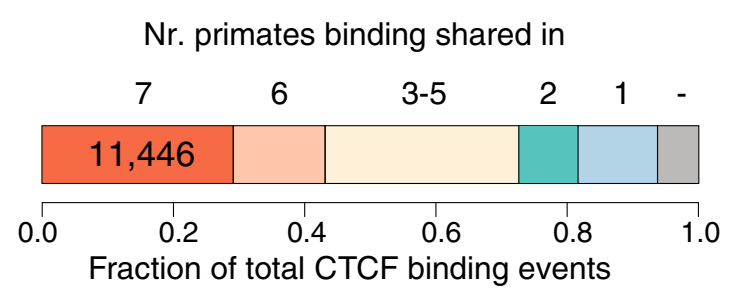

B

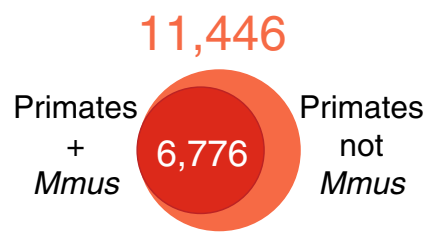

C

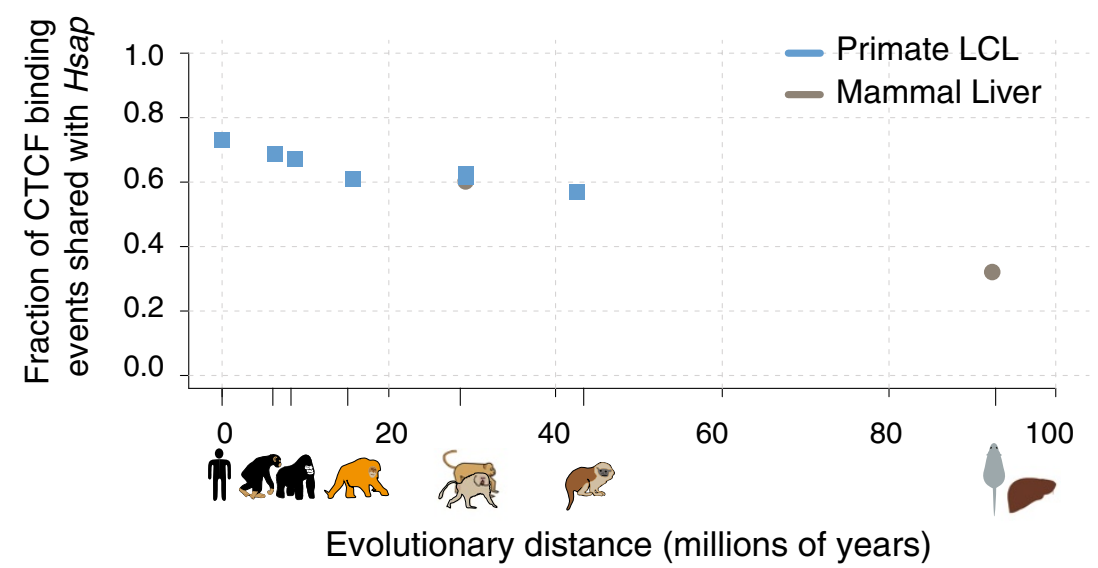

D

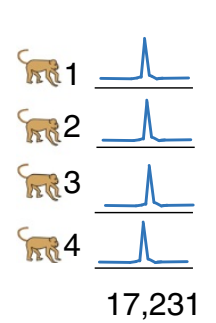

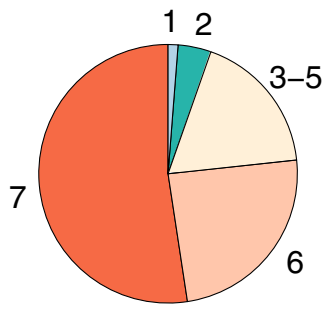
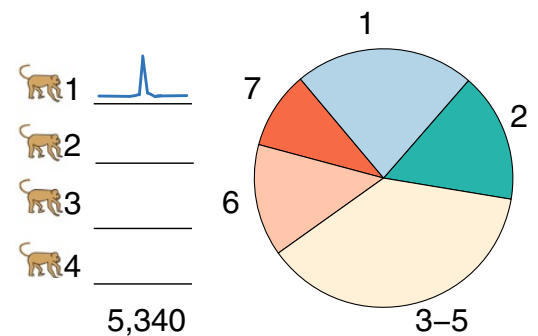

Figure 2 CTCF binding is highly conserved across primates. (A) Categorization of CTCF binding events on the basis of their evolutionary conservation. Fraction of all CTCF-bound regions across species that are not contained within the EPO multiple alignments $(-)$, present in a single species (1) and shared among two, three to five, six, or all seven primate species. (B) The fraction of primate-shared CTCF binding events (LCL) found in mouse (liver). (C) The fraction of CTCF binding events in each species overlapping human CTCF binding events. Species are ordered by evolutionary divergence time (millions of years). H.sap, P.tro, G.gor, P.pyg, M.mul, P.ham, S.oed LCL CTCF binding data (blue); H.sap M.mul and M. mus liver CTCF binding data [21] (grey). The human fraction is based on inter-individual overlaps. (D) Regions bound in four macaque cell lines (left) or one macaque cell line (right) have different patterns of conservation between species (colored as in panel $\mathbf{A}$ ).

bound regions are shared between at least two species and 11,446 binding events are shared across all analyzed primates (Figure 2A; Figure S2A,B in Additional file 1). Of the binding events common to seven primates, $98 \%$ are also bound in human liver (11,256 regions), the majority of which are also bound in mouse liver (6,776 regions; Figure 2B; Figure S2C in Additional file 1). In other words, relatively few CTCF binding events are exclusively found in primates, and not in other mammalian lineages.

The differences in CTCF binding accumulate in line with the evolutionary distance between compared species, as has been observed for more distant mammals [21], and pairwise binding overlap fractions between each primate species and human correlate negatively with evolutionary distance (Pearson's $\mathrm{r}=-0.92, P=0.004$; Figure $2 \mathrm{C}$; Figure $\mathrm{S} 2 \mathrm{~B}$ in Additional file 1). Pairwise conservation estimates are consistent with previously published comparisons of CTCF binding in rhesus macaque and mouse liver [21] as well as human and gorilla LCLs [26]. Similarly, as expected based on prior reports $[21,26]$, highly shared binding events show stronger ChIP enrichment, a better match to the consensus motif and a higher overlap with other cell types/tissues (in this case, liver) than species-specific binding events (Figure S2D-G in Additional file 1).

In order to determine the relationship between interindividual and inter-species variation, we analyzed 
independently derived LCLs from H.sap (two LCLs), P.tro (three LCLs) and M.mul (four LCLs). While over three-quarters of the regions bound by CTCF in all four probed M.mul cell lines are also shared with five or six other primates, less than one-quarter of cell line-specific bound regions show such high overlap (Figure 2D). Conversely, binding events shared across seven species are present in the vast majority (over 80\%) of the individual LCLs, in contrast to roughly half of CTCF-bound regions unique to one or two species. (Figure $\mathrm{S} 2 \mathrm{H}$ in Additional file 1). These results indicate that regions bound by CTCF across individuals are more likely to be evolutionarily conserved than individual-specific bound regions. In sum, CTCF binding that is unstable between

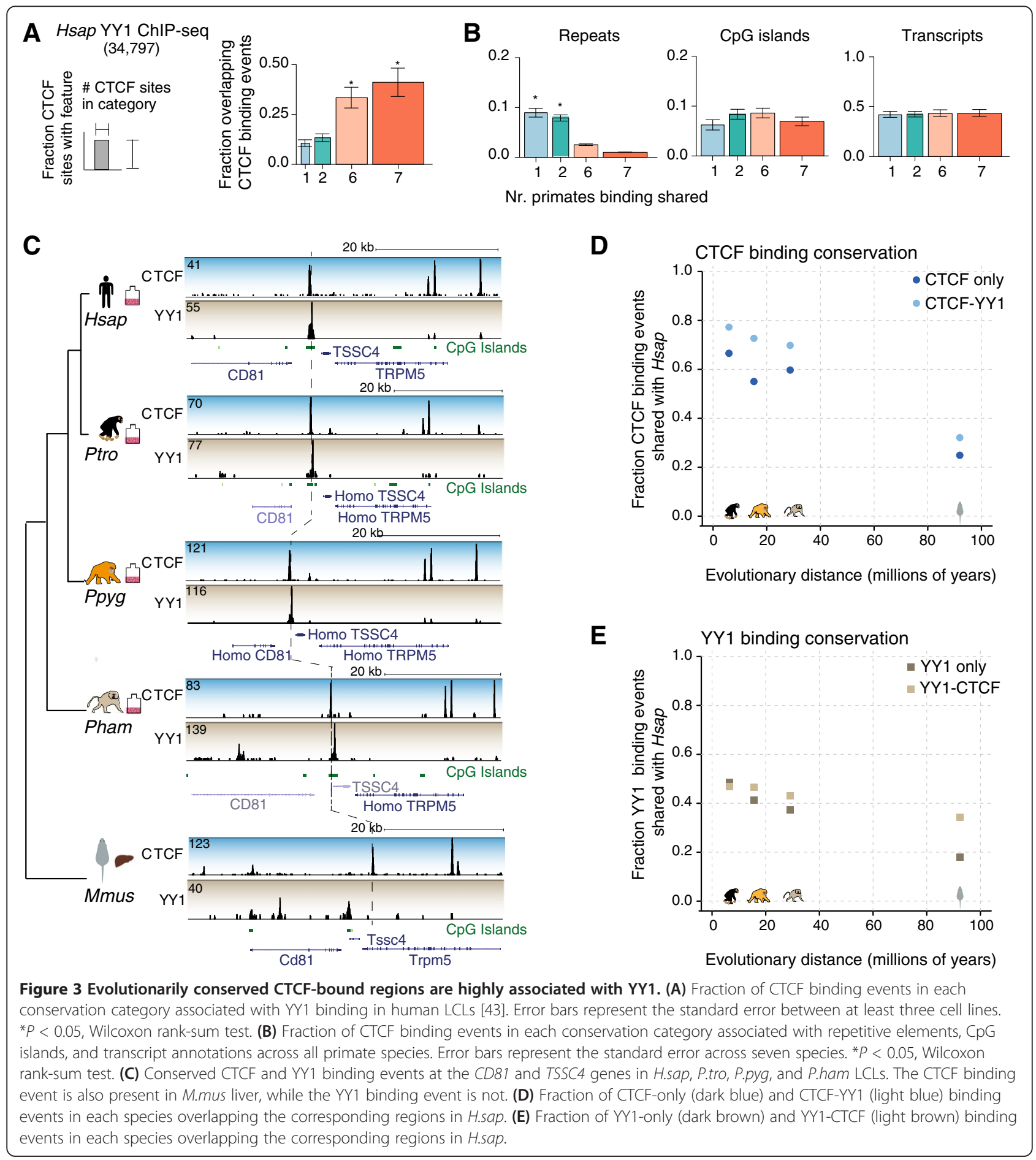


species also tends to be unstable between individuals within a species, and vice versa.

\section{Evolutionarily conserved CTCF-bound regions are co-bound by YY1}

As combinatorial binding of TFs can stabilize regulatory regions [62], and as CTCF has previously been shown to co-localize and functionally interact with the TF YY1 [42-44,51], we asked whether co-binding with YY1 could help explain high CTCF binding conservation. Indeed, we found that almost half $(41 \%)$ of the primate-shared regions are also bound by YY1 in human LCLs compared to less than $20 \%$ of species-specific CTCF binding events (Figure 3A). CTCF-YY1 co-bound regions enrich for all-primate shared CTCF binding regardless of the proximity to transcription start sites (Figure S3A in Additional file 1). In contrast, less than $15 \%$ of the regions bound by tissue-specific TFs such as NFKB and Pax 5 are also bound by CTCF, irrespective of evolutionary class (Figure S3B in Additional file 1). Evolutionarily conserved CTCF-bound regions are not specifically enriched for repetitive elements, CpG islands or transcripts (Figure 3B).

In order to establish whether YY1 co-binding stabilizes CTCF binding in evolution, we performed YY1 ChIP-seq experiments in H.sap, P.tro, P.pyg and P.ham LCLs, as well as in primary liver tissue from human and mouse. YY1 binds tens of thousands of locations in all interrogated primates, as well as in mouse liver (Figure S3C in Additional file 1). Almost 10,000 regions bound by YY1 are shared across the four primates included in this analysis (4-way shared), $61 \%$ of which are also bound in human liver and $40 \%$ of which are shared with mouse liver (Figure S3C in Additional file 1). In comparison, virtually all of the 18,000 4-way shared CTCF LCL binding events are present in human liver, and approximately $50 \%$ of these are also bound in mouse liver. In other words, for both CTCF and YY1, about half of the binding events found in multiple primate species are also bound in mouse liver. Overall, pairwise YY1 binding conservation is typically lower than observed for CTCF (Figures S2B and S3D in Additional file 1). For instance, H.sap and P.tro share $48 \%$ of YY1 binding events compared to $69 \%$ of CTCF binding events, P.tro and P.pyg 62\% YY1 versus 66\% CTCF and P.pyg and P.ham 59\% versus 71\% bound regions. Nevertheless, like CTCF, YY1 binding is more highly conserved than that observed for tissue-specific TFs such as CEBPA and HNF4A [22].

After assessing CTCF and YY1 binding independently, we combined the two datasets to analyze the stability of regions co-bound by CTCF and YY1 (Figure 3C-E). CTCF binding events that co-localize with YY1 (CTCFYY1) in one species are more likely to be shared with a second species (in this case human), and a similar effect is observed for YY1-bound regions that co-localize with
CTCF (Figure 3D,E); reflecting this, we observed stronger sequence conservation of CTCF-YY1 co-bound locations (Figure S3E in Additional file 1). For each pair of species, the regions co-bound by CTCF and YY1 are consistently more evolutionarily stable than those bound by either one of the factors in isolation.

In summary, regions co-bound by CTCF and YY1 show enhanced sequence conservation and are more likely to exist and be bound in a second mammalian species, at both shorter and wider evolutionary distances.

\section{Binding to repeat elements by either CTCF or YY1 does not explain most species-specific regions}

As both CTCF and YY1 have previously been shown to bind to and expand species-specifically via repetitive elements $[18,21,56-60]$ we analyzed the association between binding events and the repetitive genome. We found that CTCF-YY1 bound regions are less likely to overlap annotated repeats than either CTCF-only or YY1-only regions $(P<0.05$; Figure S3F in Additional file 1$)$, which is not unexpected given their high conservation. In order to determine the extent to which repetitive elements contribute to species-specific binding, we further analyzed CTCF and YY1 binding events independently.

First, we identified the annotated repetitive elements in each species bound by CTCF (Figure 4A; Figure S4A and Table S3A in Additional file 1). We detected few speciesspecific repeat associations, but observed consistent enrichments of older mammalian repeats, such as LTR41 and LTR50, as well as overrepresentation of CTCF binding to primate-specific repeats, such as LTR13 [63,64] (Figure 4B). Most were members of the LTR repeat family, consistent with previous evidence of functional exaptation of LTRs in primates (Figure S4A in Additional file 1) [65].

We also searched for repeat-specific CTCF motif words, which previously revealed CTCF-bound repeat expansions in more diverse mammalian species [21]. We detected no species-specific motif words and only a limited number of words bound at a higher frequency in primates than in other mammalian species (Figure S4B in Additional file 1). LTR13 was again identified as a CTCF-bound primatespecific repeat; however, less than 300 binding events account for this enrichment across primates, a comparatively low number considering the tens of thousands of B3-specific motif words that have shaped the CTCF binding landscape in rodent genomes [21].

We similarly identified which annotated repetitive elements are associated with YY1 binding. MLT1-type repeats of the ERVL-MaLR family, including MLT1F and MLT1J, are found to be significantly associated with YY1 binding (Figure 4C,D; Figure S4D and Table S3B in Additional file 1). MLT1-type repeats are not enriched in YY1 binding events in human and mouse livers, suggesting that the genomic interaction of YY1 and this repeat 


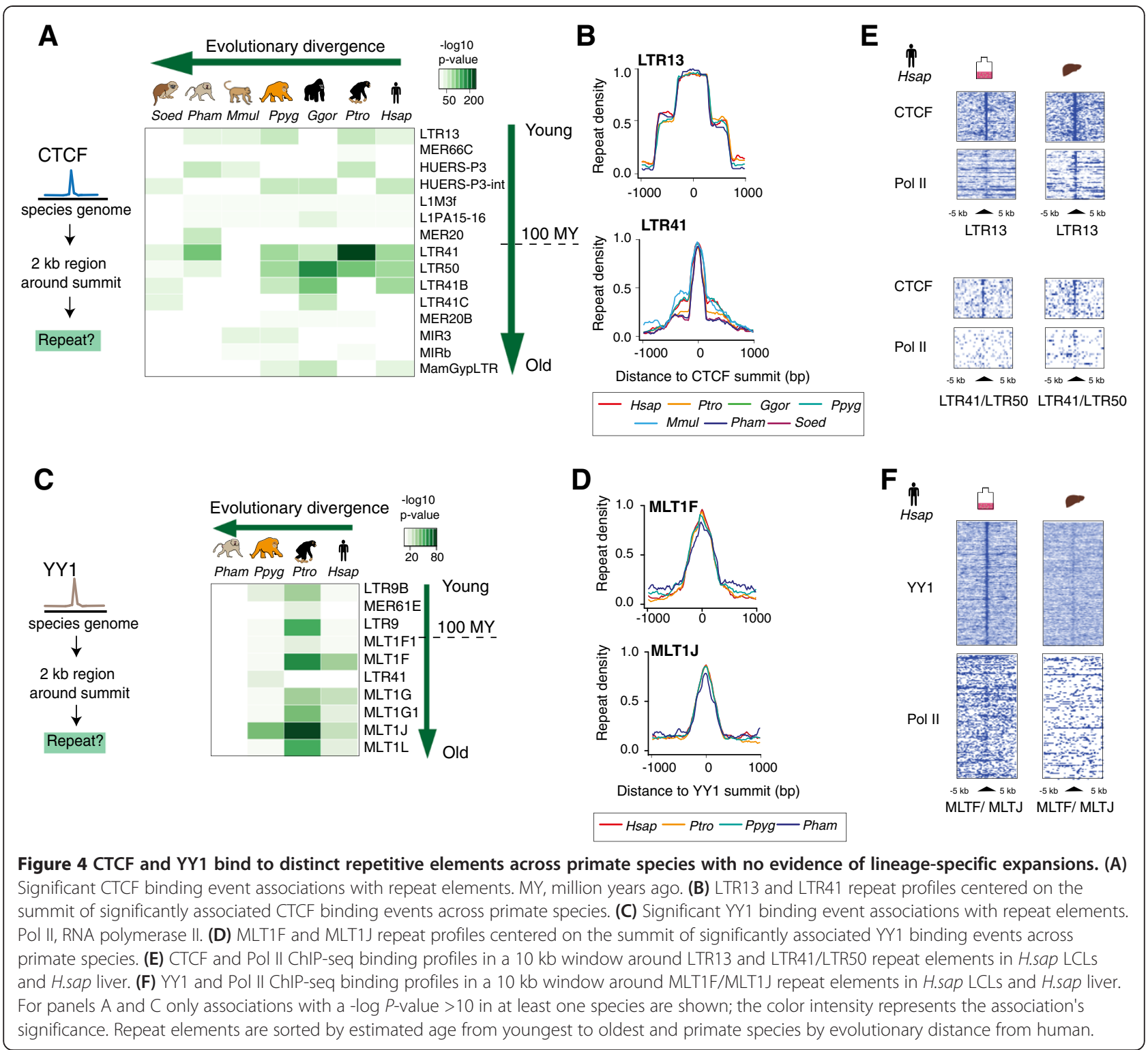

class might be tissue-specific, unlike the observed LTR repeat association with CTCF binding events (Figure 4E,F). Finally, we did not find an enrichment of repeat-embedded YY1 binding at or in close proximity to repeat-associated CTCF binding locations, indicating that these factors bind distinct repeats. Thus, repeats do not appear to be involved in CTCF-YY1 co-binding in the genome.

In sum, active repeat expansions have not substantially contributed to the CTCF binding repertoire in seven major primate lineages, and most species-specific CTCF and YY1 binding events do not appear to be mediated by repeat elements.

\section{YY1 couples CTCF binding to transcriptional activity} In primate LCLs, on average approximately one-third of CTCF-bound regions are co-bound by YY1, and nearly half of YY1-bound regions are co-bound by CTCF (Figure 5A; Figure S5A in Additional file 1). We asked whether any molecular or sequence features (aside from repeat elements) could differentiate isolated CTCF binding events from those co-bound by YY1. Overall, the binding intensities of CTCF at regions co-bound by YY1 are no greater than those of isolated CTCF binding events, suggesting that the observed pattern is not simply driven by ChIP enrichment class $(P>0.05$; Figure S5B in Additional file 1). De novo motif discovery identified the canonical motifs for both CTCF and YY1 at shared CTCF-YY1 bound locations, indicating that both factors directly bind to DNA in general (Figure S5C in Additional file 1). However, we did not observe a consistent spacing constraint between the two motifs at co-bound regions (data not shown). Importantly, we found CTCF-YY1 co-bound 


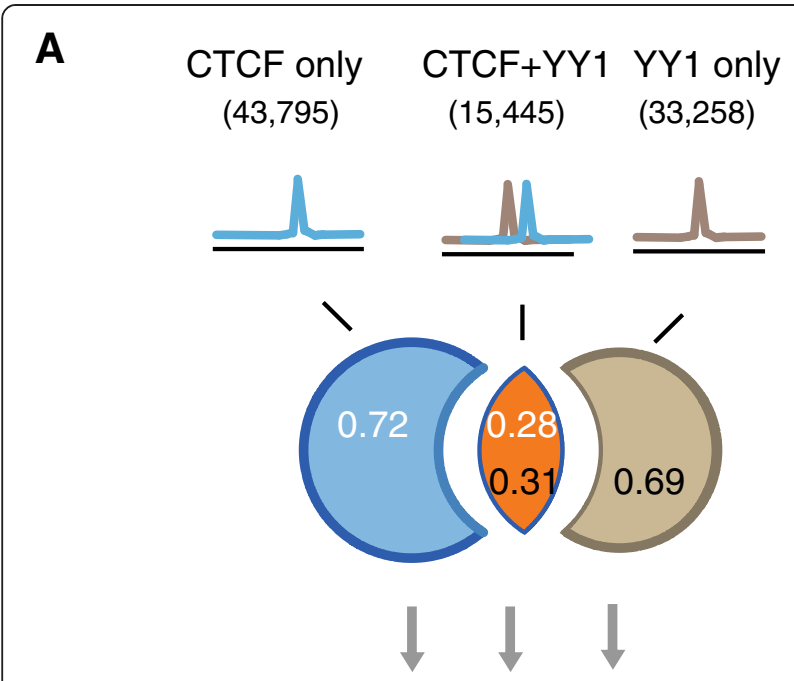

B

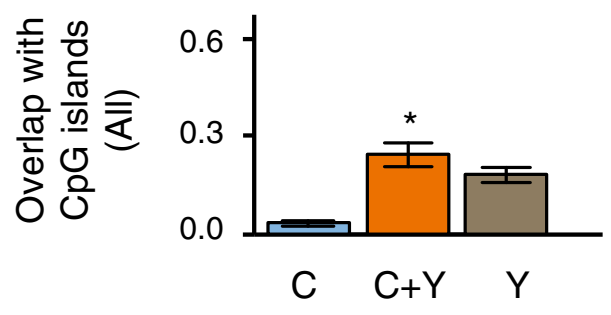

C

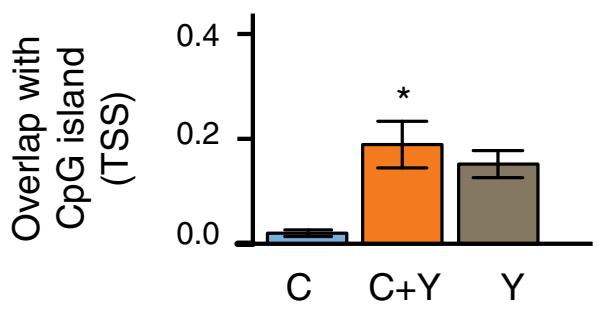

Figure 5 CTCF-YY1 co-bound regions associate with CpG islands. (A) The fraction of overlapping CTCF- and YY1-bound regions in H.sap, P.tro, P.pyg, and P.ham LCLs. (B) The fraction of CTCF-only, CTCF-YY1 and YY1-only binding events overlapping CpG islands across species. (C) The fraction of CTCF-only (C), CTCF-YY1 $(C+Y)$ and $Y Y 1$-only $(Y)$ binding events overlapping $C p G$ island transcription start sites (TSS) across species. Error bars represent standard error of the mean among four species. ${ }^{*} P<0.05$, Wilcoxon rank-sum test, $C$ versus $C+Y$.

regions to be significantly more associated with CpG islands $(P<0.05)$ and $\mathrm{CpG}$ island promoters $(P<0.05)$ than isolated CTCF binding events, indicating that co-bound regions may be more transcriptionally active (Figure 5B,C; Figure S5D in Additional file 1).

To further explore the relationship between transcription and conservation of CTCF-YY1 co-bound regions, we analyzed the ChIP-seq data from mouse and human liver with corresponding functional data for basal transcriptional machinery, tissue-specific TFs, and histone marks $[22,66]$. We found that CTCF-YY1 co-bound regions overlap marks of transcriptional activity, including RNA polymerase II (RNA Pol II), the active H3K4me3 histone modification, as well as liver-specific transcriptional regulators such as HNF4A and CEBPA (Figure 6A,B; Figure S6A,B in Additional file 1). In contrast, CTCF-bound regions lacking YY1 (CTCF-only) rarely co-localize with marks of active transcription and tissue-specific TF binding. CTCF-YY1 co-bound regions in liver tend to be associated with core liver functions such as lipid metabolism and transport in both human and mouse (Figure S6C in Additional file 1).

In order to determine whether the presence of $\mathrm{YY} 1$ at CTCF-bound regions has an effect on transcriptional output, the expression of genes bound by only CTCF versus those bound by CTCF and YY1 were compared. Genes overlapping YY1 binding events (including YY1only and YY1-CTCF) are significantly more highly expressed than are genes overlapping CTCF-only binding events $\left(P<10^{-16}\right)$ in both human and mouse liver (Figure 6C; Figure S6D in Additional file 1).

In sum, CTCF-YY1 co-bound regions are functionally similar to YY1-only regions based on their association with increased gene expression and enrichment of Pol II, H3K4me3 and liver-specific transcriptional regulators. This means that the CTCF occupied regions co-bound by YY1 show not only stronger evolutionary stability, but also increased transcriptional activity, in contrast to the bulk of CTCF-bound regions.

\section{Discussion}

Our mapping and inter-species comparison of CTCF binding in cell lines from H.sap, P.tro, G.gor, P.pyg, M.mul, P.ham and S.oed has revealed over 11,000 genomic locations bound by CTCF across primates, consistent with the high conservation of CTCF binding observed in more distant mammalian species $[19,21,67]$. This estimate was obtained despite the fact that our analytical approach did not assume that CTCF binding is conserved, and as such is likely to underestimate true inter-species overlaps $[21,22]$. In contrast, other related studies have assumed conservation and minimized species-specific differences by using a dual cutoff, anchored in a single reference species $[34,68]$.

Despite our conservative approach, we found that $60 \%$ of CTCF-bound regions are shared between H.sap and M.mul, whereas a recent comparison across 25 million years of Drosophila sp. evolution, a similar divergence time [69], has revealed approximately $30 \%$ binding conservation [70]. This discrepancy could be caused by differences in CTCF function between the chordate and arthropod phyla: CTCF is the only well-characterized insulator-binding protein in vertebrates, where it seems to be largely responsible for three-dimensional genome 


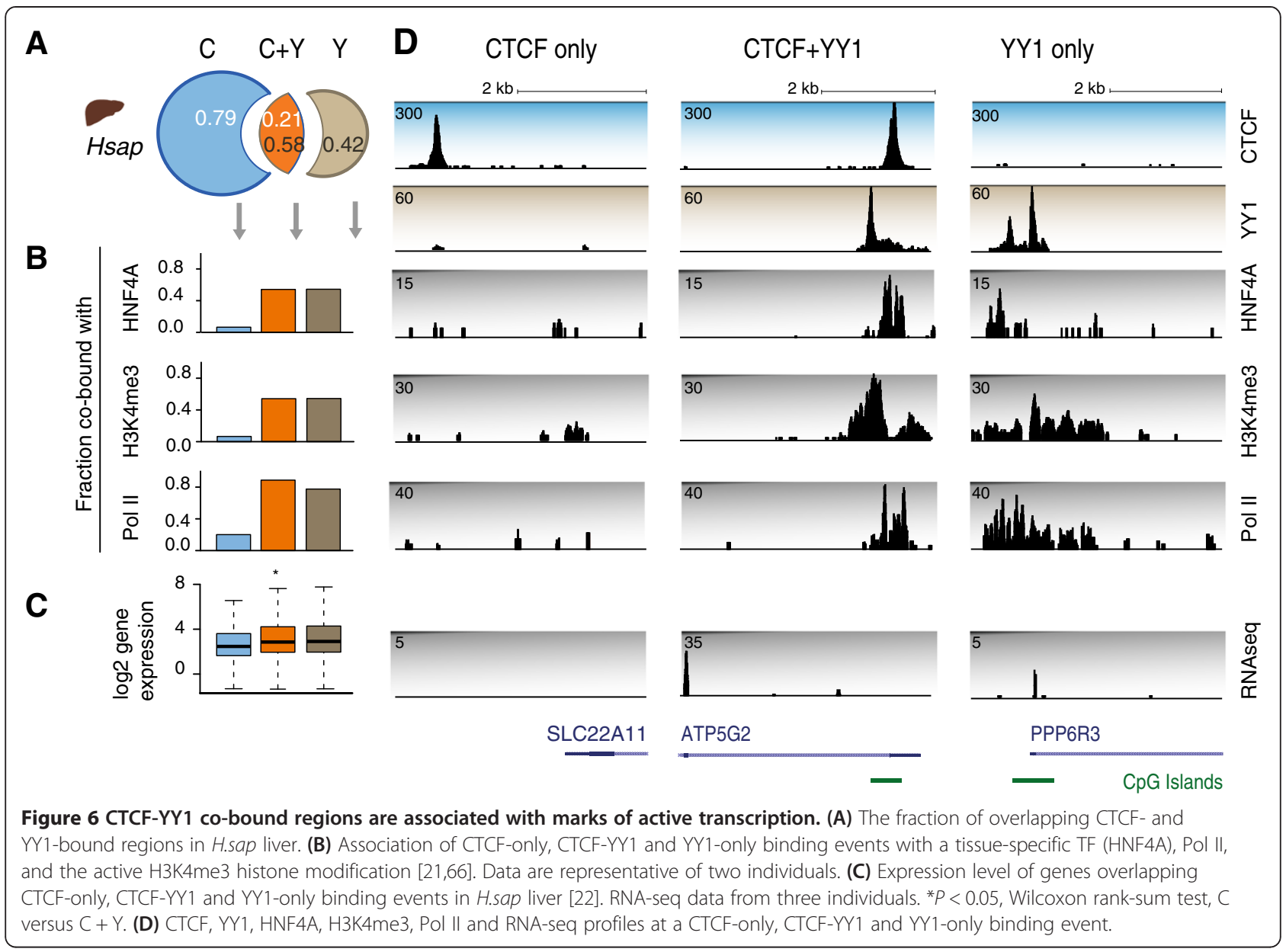

organization, as well as regulating various transcriptional and gene regulatory processes in collaboration with cohesin [71]. In contrast, Drosophila sp. have multiple insulator proteins and thus may place lower constraint on CTCF binding sites.

Previous studies have shown that the expansion of repetitive elements appears to be a major mechanism by which CTCF increases its target landscape in individual mammalian lineages $[18,21,72]$. Here, we identified particular repeat types associated with CTCF binding across primates, some of which have previously been shown to associate with CTCF in human, including LTR41 in human embryonic stem cells [18] and LTR13 across multiple cell types $[18,63]$. However, we did not find systematic evidence for a repeat-mediated expansion of CTCF binding in primate clades. This comparative quiescence of repeats that carry CTCF binding sequences in primates relative to rodents might be in part due to differences in genome transposon content and activity. For instance, mice have more lineage-specific repeat elements than humans, as well as greater transposon activity and fewer ancestral repeats $[73,74]$.

\section{Conclusions}

In searching for factors contributing to the conservation of CTCF binding we discovered that co-binding by YY1 is an ancient regulatory mechanism that appears to increase the evolutionary stability of CTCF binding in multiple mammalian species. CTCF and YY1 have previously been shown to co-localize and physically interact $[9,42,43,46,75]$ but their combined, genome-wide interaction has not been investigated across multiple species.

Our analysis revealed that one mechanism stabilizing the protein-DNA contacts in regions co-bound by CTCF and YY1 may be their association with active chromatin and gene expression (previously reported for CTCF in [10,76-78]). It has been shown that CTCF can interact directly with Pol II and target it to a subset of CTCF sites genome-wide [79]. Our data now reveal that this likely occurs in the presence of YY1, because in its absence, CTCF-bound regions almost never co-localize with Pol II, H3K4me3, or tissue-specific TFs, whether proximal or distal to genes. These observations suggest that with respect to transcriptional activity, YY1 is the functionally dominant factor at co-bound locations. 
Experiments performed at a single genetic locus have shown that CTCF can form a complex with YY1 and the tissue-specific factor Oct4 that binds Tsix and Xite to control X-chromosome pairing and counting in embryonic stem cells [80]. Co-binding of CTCF and YY1 thus appears to indicate globally to the chromatin remodeling machinery which euchromatic regions are to be activated [81]. The integration of these discoveries suggests a model wherein co-transcriptional activity of YY1bound regions may help conserve CTCF and YY1 binding via functional deployment.

In summary, CTCF-YY1 co-bound regions are not only preferentially and highly conserved but also show hallmarks of transcriptional function that could provide selective pressure to preserve specific protein-DNA contacts across millions of years of mammalian evolution.

\section{Materials and methods}

\section{Cell line material}

Lymphoblast cell lines were obtained for seven primate species. The species, cell line and source are shown in Table S1 in Additional file 1. All cell lines were transformed by the Epstein-Barr virus except for the M.mul cell lines, which were transformed by Herpesvirus papio.

Cells were grown in suspension at a confluency of 200,000 cells $/ \mathrm{ml}$ to $1 \times 10^{6}$ cells $/ \mathrm{ml}$ in RPMI1640 media supplemented with $15 \%$ fetal bovine serum and $2 \mathrm{mM}$ L-glutamine, $100 \mathrm{I} . \mathrm{U} / \mathrm{ml}$ penicillin and $100 \mu \mathrm{g} / \mathrm{ml}$ streptomycin. We cross-linked $1 \times 10^{8}$ cells with $1 \%$ formaldehyde as previously described [82].

\section{Tissue material}

\section{Mouse}

C57BL/6 J mice were housed in the Biological Resources Unit under UK Home Office licensing. Tissue was obtained from at least two independent males and formaldehyde cross-linked as described in [82].

\section{Human}

Male and female human tissue samples were obtained from biopsied tissue collected at Addenbrooke's Hospital, Cambridge, and provided by the Biobank under human tissue license 08/H0308/117. Liver tissue was also obtained from the Liver Tissue Distribution Program (NIDDK contract number N01-DK-9-2310) at the University of Pittsburgh.

\section{ChIP-seq}

ChIP-seq assays were performed as previously described [82]. Protein-bound DNA was immunoprecipitated with antibodies against CTCF (Millipore, Billerica, MA, USA, 07-729), or YY1 (Santa Cruz Biotechnology, Dallas, TX, USA, sc-281). Immunoprecipitated DNA was endrepaired, A-tailed and ligated to single-end Illumina sequencing adapters before 18 cycles of PCR amplification. DNA fragments (200 to $300 \mathrm{bp}$ ) were selected and 36 bp reads sequenced on an Illumina Genome Analyser II according to the manufacturer's instructions.

\section{Published ChIP-seq experiments}

The following published ChIP-seq data were used: mouse liver CTCF [21], mouse liver H3K4me3 and Pol II [83], human H3K4me3 and Pol II [66], human and mouse liver HNF4A and CEBPA [22]. ENCODE data used were from the following cell lines: for CTCF, GM12878, GM12891, GM12892, GM19239, GM19240, HepG2, H1-hESC; for YY1, GM12878, GM12891, GM12892, HepG2, H1-hESC; for NFKB, GM12878 (no treatment), GM12891 (tumor necrosis factor alpha treatment), GM12892 (tumor necrosis factor alpha treatment); for Pax5, GM12878, GM12891, GM12892; and for Pol II, GM12878 [43]. Published mouse embryonic stem cell ChIP-seq data for CTCF and YY1 were also used $[19,84])$.

\section{Computational methods}

All computational analyses were performed with scripts written in Perl, Bioperl 1.2.3, and R version 2.11.1, using packages available in Bioconductor 2.6 (Additional file 2). Displayed error bars represent the standard error of the mean and significance levels were estimated using onesided Wilcoxon rank-sum tests if not otherwise stated.

\section{Read alignment and peak-calling}

ChIP and input sequencing reads from all LCL datasets were aligned using Bowtie [85] 0.12.7 with the parameters '-n 2 -m 3 -k 1 -best' to the following genome assemblies: human GRCh37, chimpanzee CHIMP2.1, gorilla gorGor3, orangutan PPYG2, macaque Mmul 1, and marmoset $\mathrm{C}$. jacchus 3.2.1. All sequence, genome annotations (genes, transcripts, CpG islands) and comparative genomics data were taken from Ensembl release 60. Repeat element annotation was downloaded from the UCSC Table Browser for all species. The baboon data were aligned to the macaque genome, as it was the closest fully assembled genome. When available (all species except for marmoset), only chromosomes and not unmapped contigs were used. Aligned reads were filtered for duplicates, uncalled bases (a maximum of three Ns were allowed) and low complexity reads. Regions of high ChIP enrichment (peaks/bound regions/binding events) were detected with CCAT 3.0 [86] on individual replicates using the parameters 'fragmentSize 100, slidingWinSize 150, movingStep 10, isStrandSensitiveMode 1 , minCount 10, minScore 4.0, bootstrapPass 50' for the two TFs and 'fragmentSize 200, slidingWinSize 100, movingStep 20, isStrandSensitiveMode 0, minCount 10, minScore 4.0, bootstrapPass 50' for Pol II. Naked DNA (input) was used as control and the FDR cutoff was set 
to 0.1. Peaks were then merged among replicates in each organism by taking the intersection and additionally adding replicate-unique peaks with an FDR $<0.05$. The similarity between individual replicates was assessed by calculating the correlation (Spearman's rho) between read counts inside peak regions (Figure S1 in Additional file 1).

\section{ChIP-seq data visualization}

ChIP-seq data from each species was visualized on the corresponding species genome on the UCSC genome browser [87]. Human (GRCh37/hg19), chimpanzee (CGSC 2.1/panTro2), gorilla (gorGor3.1/gorGor3), orangutan (WUGSC 2.0.2/ponAbe2), macaque and baboon (MGSC Merged 1.0/rheMac2), tamarin (WUGSC 2.0.2/calJac1), and mouse (NCBI37/mm9).

\section{Conservation analysis}

We performed all our inter-species comparisons based on the 6-primate EPO (PrimateEPO) and the 11-way eutherian mammals (EPO) multiple sequence alignments (MSAs) available in Ensembl Compara release 60. Binding events discovered by CCAT at an FDR of 0.05 were projected onto all study species using the MSA through the Ensembl Compara Application Programming Interface (API). We restricted the evolutionary analysis to regions of the genome included in the MSA. Each of the study species was used as anchor species, and the region of interest projected onto the other species. In order to determine the degree of commonality between the species, projections were then overlapped ( $\geq 1 \mathrm{bp}$ ) with binding events - that is, binding events called at an FDR of 0.05 in one species that overlap with binding events called at the same FDR in a second species are called shared. To estimate the fraction of putatively shared binding events missed by this approach, we fixed the FDR in one species (human), varied it in the other six species (up to FDR $=0.5$ ) and calculated the new percentage overlaps (data not shown). Conservation estimates increased by less than 10\% compared to the fixed values reported in the manuscript, suggesting that while the method employed here does underestimate conservation levels, this effect is limited.

Overlap numbers differed by up to tens of bound regions depending on which species was used for anchoring. The percentage overlap numbers reported in Figure 2C, Figure S2B in Additional file 1, Figure 3D-E, and Figure S3D in Additional file 1 are averages between the two analysis directions (for example, shared humanchimpanzee regions from human and chimpanzee perspective). The human-human overlap percentage was obtained by calculating the overlap fraction of our operative peak set with different LCLs when available: five different cell lines for human (ENCODE LCL GM12878, GM12891, GM12892, GM19239, GM19239, GM19240), three different LCLs for chimpanzee and four different LCLs for rhesus macaque. The median value is displayed in Figure $2 \mathrm{C}$ based on primate (blue square) and mammalian (grey circle) alignments. Evolutionary time between the species was obtained from [88] (median).

For the comparative analyses displayed in Figures 2 and 3 and Figures S2 and S3 in Additional file 1 we divided the bound regions into six different categories in each of the seven primate species (we refer to these categories as 'conservation classes'): (i) species-specific and not included in the genome-wide multiple alignments, (ii) species-specific and included in the alignments, (iii) shared between two species only, (iv) shared among three to five species, (v) shared among six species, and (vi) shared among all seven analyzed primates. We calculated the relative fraction of bound regions belonging to these six categories and displayed them as barplots in Figure S2A in Additional file 1. The median values across all seven species are shown in Figure 2A as well as the number of seven-way shared peaks (based on the human genome).

For the sequence conservation analysis of CTCF-only and CTCF-YY1 regions in Figure S3E in Additional file 1, the Phastcons tool [89] in Galaxy Cistrome [90] was used.

\section{Properties of different peak categories and CTCF-YY1 bind- ing event classes}

Four peak categories (species-specific (1), shared between exactly two species (2), shared among exactly six species (6) and shared among all seven species (7)) were further analyzed for diverse properties in each single species: CCAT score (proportional to ChIP enrichment), the top NestedMica motif match score distribution (with 0 corresponding to the consensus motif), the numbers of peaks with at least one motif, overlaps with peaks called in distinct LCL cell lines when available (human, two; chimpanzee, three; macaque, four), overlaps with transcripts, CpG islands, repetitive elements, Pol II, and publicly available TF binding data from ENCODE. Barplot widths are proportional to the number of regions belonging to each category. We also performed a detailed conservation-inter-individual overlap analysis using four distinct rhesus macaque LCL lines. We selected two types of CTCF-bound regions: (1) bound in only one of the four cell lines and (2) bound in all four cell lines. We then asked how often these regions were shared with the other species and displayed the relative fractions as pie charts in Figure 2D.

Three distinct classes of CTCF/YY1 bound regions (CTCF-only, CTCF-YY1 and YY1-only regions) were analyzed for their properties, using data from four primate LCLs (human, chimpanzee, orangutan, and baboon), as well as human and mouse liver data. Additionally, previously published Pol II, H3K4me3, CEBPA, and HNF4A 
data in human and mouse liver were intersected with the three classes of bound regions.

\section{Repeat element association}

We tested genome-wide association of annotated repeat elements with LCL CTCF/YY1 binding events in each single species by using a binomial test. We estimated background probabilities from median overlaps of repeat elements with randomized CTCF/YY1 binding events, and corrected for multiple testing by the BenjaminiHochberg method. Repeats that obtained a $P$-value $\leq 0.01$ are included in Figure S4A,D in Additional file 1; repeats with a $-\log P$-value $>10$ in at least one species are displayed in Figure 4 and Table S3 in Additional file 1.

We estimated the repeat divergence from the consensus sequence based on the number of substitutions from the consensus ('milliDiv' column in the UCSC-obtained RepeatMasker tracks) and the age of individual bound repeat elements by dividing the substitution number by the mutation rate estimated for mammalian species $\left(2.2 \times 10^{9}\right.$ per base pair per year) [91] and rodents $(4.5 \times$ $10^{9}$ per base pair per year) [74]. Repeat ages were used to order the heatmaps shown in Figure 4. Repeats were sorted by class in Figure S4 in Additional file 1 [92]. Repeat profile plots centered on CTCF and YY1 peak summits in human LCLs were displayed for the top two enriched repeats, LTR13/LTR41, and MLT1J/MLT1F, respectively.

We also performed a detailed motif-word analysis as described in [21]. Individual motif instances obtained by scanning the genomes with the CTCF position weight matrix (PWM) were collected as DNA motif words (14mers). We defined the set of bound words as the union of words falling inside bound regions in our study species. We counted individual occurrences of all motif words in the studied species, and divided by a normalization factor, proportional to the total number of bound bases in a certain species, obtaining a normalized occurrence (nocc) measure for each word and species:

$$
\text { nocci } ; j=\text { nocci } ; j / \text { factor }
$$

where nocc is the word count, $i$ is the word number, $j$ is the species number, and factor is defined as the total bound bases divided by $1,000,000$. We selected only words that occurred at least five times in at least one species. We used these normalized word occurrence values to define species-specific words as follows:

$$
\text { normWord }=\log 2((\operatorname{nocc}(S)+1)=\max (\operatorname{nocc}(R)+1))
$$

where $S$ is the species of interest and $R$ all other species or all other species from a different branch of the evolutionary tree (considered groups were hominidae, Old World monkeys, New World monkeys, primates, and non-primate mammals). We fitted a normal distribution to normWord and chose a cutoff that corresponded to a FDR of 0.05 after multiple testing correction. All words with nocc(S) greater than the determined cutoff were selected for each species. For these selected words, we counted the number of CTCF-bound sequences of this type that are located inside annotated repeat elements. We display the log number of such words, as well as the analogous results obtained in mouse livers (for comparison) in Figure S4B in Additional file 1.

Repeat read profiles displayed in Figure 4E,F were generated by quantifying the read counts in 200 windows of 50 bp each centered around CTCF or YY1 peak summits that were contained in the repeat classes of interest. The obtained matrices were then visualized in Java TreeView while keeping the scale the same for each dataset [93].

\section{Motif analysis}

Motif discovery was conducted with NestedMica [94] using the parameters '-minLength 5 -maxLength 30 -numMotifs 6' and a fourth order background model trained on mammalian regulatory regions (DHS) data. Discovered motifs were confirmed using MEME [95], with the options '-nmotifs 5 -minsites 100 -minw 6 -maxw 25 -revcomp - maxsize 500000 -dna'. We selected the top 1,000 peaks ordered by CCAT score and used 25 bp upand downstream of the peak summit as input for motif discovery. As the obtained top motifs were virtually identical in all studied species, we merged them into a single PWM that we used in further motif analysis steps. NestedMica's nmscan with a cutoff of -15 was used for motif matching (a score of 0 corresponds to a perfect match to the motif consensus) displayed in Figure S5C in Additional file 1 are obtained from all sequences that match the CTCF and YY1 PWMs inside regions positive for both CTCF and YY1 ChIP signal.

\section{Functional association analysis}

CTCF regions co-bound with YY1 were analyzed relative to all CTCF-bound regions in Figure S6C in Additional file 1 to determine whether these regions were associated with common biological pathways using cPath within the GREAT bioinformatic tool $[96,97]$.

\section{Expression analysis}

We used published liver RNA-seq data in human and mouse to test the association between YY1/CTCF binding events with transcriptional activity [22]. Reads were mapped to Ensembl release 60 transcript annotation and transcript levels quantified using mmseq [98]. We compared $\log 2$ (transcript estimates) for transcripts overlapping YY1-only, CTCF-only, or at least one CTCF-YY1 binding event using a Wilcoxon signed-rank test and 
display the data as boxplots in Figure $6 \mathrm{C}$ and Figure S6D in Additional file 1.

\section{Data access}

CTCF and YY1 ChIP-seq data have been deposited under Arrayexpress, accession number E-MTAB-1511.

\section{Additional files}

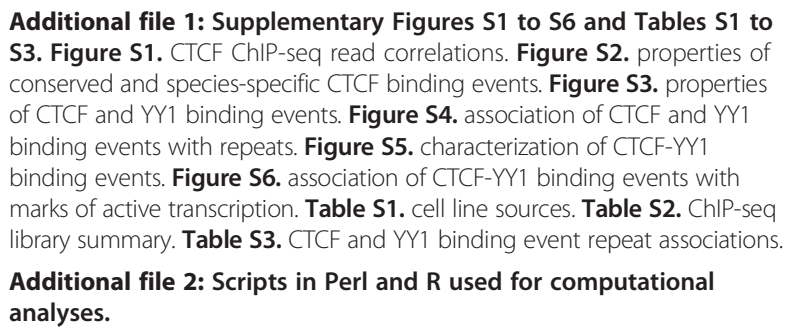

\section{Abbreviations}

bp: base pair; C.jac: Callithrix jacchus; ChIP: chromatin immunoprecipitation; FDR: false discovery rate; G.gor: Gorilla gorilla; H.sap: Homo sapiens;

LCL: Iymphoblastoid cell line; M.mul: Macaca mulatta; MSA: multiple sequence alignment; P.ham: Papio hamadryas; P.pyg: Pongo pygmaeus; P.tro: Pan troglodytes; Pol II: RNA polymerase II; PWM: position weight matrix; S.oed: Sanguinus oedipus; TF: transcription factor.

\section{Competing interests}

The authors declare that they have no competing interests.

\section{Authors' contributions}

PCS, MCW, DTO, and PF designed and conceived experiments. PCS, MCW and AJF analyzed data. MCW performed experiments. CEC cultured and cross-linked the macaque cell lines. PCS, MCW, DTO, and PF wrote the manuscript with input from all authors. YG, DTO, and PF oversaw the work. All authors read and approved the final manuscript.

\section{Acknowledgements}

We are grateful to the Cambridge Institute Genomics and Bioinformatics Core facilities and Margus Lukk and Tim F Rayner for computational support. Thank you to the Health Protection Agency Cell culture collections and the ECACC for culturing and cross-linking the 81.3, EB176(JC), EB(JC), EB185(JC), 26 CB1, and B95-8 cell lines. We thank the New England Primate Research Center for the rhesus macaque LCLs and Chris Tyler-Smith for the GG013 gorilla LCL. This research was supported by: ERC Starting Grant and EMBO Young Investigator Award (DTO); Wellcome Trust Awards WT095908 (PF) and WT098051 (PF, DTO); NIH Grant GM084996 (YG); University of Cambridge (PCS, MCW, AJF, DTO); EMBL (PCS, AJF, PF); Cancer Research UK (MCW, DTO); Commonwealth Scholarship Commission (MCW); EMBO Short Term Fellowship (MCW).

\footnotetext{
Author details

'European Molecular Biology Laboratory, European Bioinformatics Institute, Wellcome Trust Genome Campus, Hinxton, Cambridge CB10 1SD, UK. ${ }^{2}$ University of Cambridge, Cancer Research UK-Cambridge Institute, Robinson Way, Cambridge CB2 ORE, UK. ${ }^{3}$ Current address: Department of Human Genetics, University of Chicago, Chicago, IL 60637, USA. "Wellcome Trust Sanger Institute, Wellcome Trust Genome Campus, Hinxton CB10 1SA, UK. ${ }^{5}$ Current address: Laboratory of Systems Biology and Genetics, Institute of Bioengineering, School of Life Sciences, École Polytechnique Fédérale de Lausanne (EPFL), Lausanne CH-1015, Switzerland.
}

Received: 13 November 2013 Accepted: 31 December 2013

Published: 31 December 2013

\section{References}

1. Klenova EM, Nicolas RH, Paterson HF, Carne AF, Heath CM, Goodwin GH, Neiman PE, Lobanenkov W: CTCF, a conserved nuclear factor required for optimal transcriptional activity of the chicken c-myc gene, is an 11-Zn-finger protein differentially expressed in multiple forms. Mol Cell Biol 1993, 13:7612-7624.

2. Moon H, Filippova G, Loukinov D, Pugacheva E, Chen Q, Smith ST, Munhall A, Grewe B, Bartkuhn M, Arnold R, Burke LJ, Renkawitz-Pohl R, Ohlsson R, Zhou J, Renkawitz R, Lobanenkov V: CTCF is conserved from Drosophila to humans and confers enhancer blocking of the Fab-8 insulator. EMBO Rep 2005, 6:165-170

3. Baniahmad A, Steiner C, Kohne AC, Renkawitz R: Modular structure of a chicken lysozyme silencer: involvement of an unusual thyroid hormone receptor binding site. Cell 1990, 61:505-514.

4. Lobanenkov W, Nicolas RH, Adler W, Paterson H, Klenova EM, Polotskaja AV, Goodwin GH: A novel sequence-specific DNA binding protein which interacts with three regularly spaced direct repeats of the CCCTC-motif in the 5'-flanking sequence of the chicken c-myc gene. Oncogene 1990, 5:1743-1753.

5. Vostrov AA, Quitschke WW: The zinc finger protein CTCF binds to the APBbeta domain of the amyloid beta-protein precursor promoter. Evidence for a role in transcriptional activation. J Biol Chem 1997, 272:33353-33359.

6. Bell AC, West AG, Felsenfeld $G$ : The protein CTCF is required for the enhancer blocking activity of vertebrate insulators. Cell 1999, 98:387-396.

7. Hark AT, Schoenherr CJ, Katz DJ, Ingram RS, Levorse JM, Tilghman SM: CTCF mediates methylation-sensitive enhancer-blocking activity at the H19/lgf2 locus. Nature 2000, 405:486-489.

8. Shukla S, Kavak E, Gregory M, Imashimizu M, Shutinoski B, Kashlev M, Oberdoerffer P, Sandberg R, Oberdoerffer S: CTCF-promoted RNA polymerase II pausing links DNA methylation to splicing. Nature 2011, 479:74-79.

9. Donohoe ME, Zhang LF, XU N, Shi Y, Lee JT: Identification of a Ctcf cofactor, Yy1, for the X chromosome binary switch. Mol Cell 2007, 25:43-56.

10. Chen $H$, Tian $Y$, Shu W, Bo X, Wang S: Comprehensive identification and annotation of cell type-specific and ubiquitous CTCF-binding sites in the human genome. PLoS One 2012, 7:e41374.

11. Cuddapah S, Jothi R, Schones DE, Roh TY, Cui K, Zhao K: Global analysis of the insulator binding protein CTCF in chromatin barrier regions reveals demarcation of active and repressive domains. Genome Res 2009, 19:24-32.

12. Kim TH, Abdullaev ZK, Smith AD, Ching KA, Loukinov DI, Green RD, Zhang MQ, Lobanenkov W, Ren B: Analysis of the vertebrate insulator protein CTCF-binding sites in the human genome. Cell 2007, 128:1231-1245.

13. Fu Y, Sinha M, Peterson CL, Weng Z: The insulator binding protein CTCF positions 20 nucleosomes around its binding sites across the human genome. PLoS Genet 2008, 4:e1000138.

14. Handoko L, Xu H, Li G, Ngan CY, Chew E, Schnapp M, Lee CW, Ye C, Ping $J$, Mulawadi F, Wong E, Sheng J, Zhang Y, Poh T, Chan CS, Kunarso G, Shahab A, Bourque G, Cacheux-Rataboul V, Sung WK, Ruan Y, Wei CL: CTCF-mediated functional chromatin interactome in pluripotent cells. Nat Genet 2011, 43:630-638.

15. Wang H, Maurano MT, Qu H, Varley KE, Gertz J, Pauli F, Lee K, Canfield T, Weaver M, Sandstrom R, Thurman RE, Kaul R, Myers RM, Stamatoyannopoulos JA: Widespread plasticity in CTCF occupancy linked to DNA methylation. Genome Res 2012, 22:1680-1688.

16. Shen Y, Yue F, McCleary DF, Ye Z, Edsall L, Kuan S, Wagner U, Dixon J, Lee L, Lobanenkov W, Ren B: A map of the cis-regulatory sequences in the mouse genome. Nature 2012, 488:116-120.

17. Borneman AR, Gianoulis TA, Zhang ZD, Yu H, Rozowsky J, Seringhaus MR, Wang LY, Gerstein M, Snyder M: Divergence of transcription factor binding sites across related yeast species. Science 2007, 317:815-819.

18. Kunarso G, Chia NY, Jeyakani J, Hwang C, Lu X, Chan YS, Ng HH, Bourque G: Transposable elements have rewired the core regulatory network of human embryonic stem cells. Nat Genet 2010, 42:631-634.

19. Martin D, Pantoja C, Fernandez Minan A, Valdes-Quezada C, Molto E, Matesanz F, Bogdanovic O, de la Calle-Mustienes E, Dominguez O, Taher $L$ Furlan-Magaril M, Alcina A, Canon S, Fedetz M, Blasco MA, Pereira PS, Ovcharenko I, Recillas-Targa F, Montoliu L, Manzanares M, Guigo R, Serrano M, Casares F, Gomez-Skarmeta JL: Genome-wide CTCF distribution in 
vertebrates defines equivalent sites that aid the identification of disease-associated genes. Nat Struct Mol Biol 2011, 18:708-714.

20. Odom DT, Dowell RD, Jacobsen ES, Gordon W, Danford TW, Maclsaac KD, Rolfe PA, Conboy CM, Gifford DK, Fraenkel E: Tissue-specific transcriptional regulation has diverged significantly between human and mouse. Nat Genet 2007, 39:730-732.

21. Schmidt D, Schwalie PC, Wilson MD, Ballester B, Goncalves A, Kutter C, Brown GD, Marshall A, Flicek P, Odom DT: Waves of retrotransposon expansion remodel genome organization and CTCF binding in multiple mammalian lineages. Cell 2012, 148:335-348.

22. Schmidt D, Wilson MD, Ballester B, Schwalie PC, Brown GD, Marshall A Kutter C, Watt S, Martinez-Jimenez CP, Mackay S, Talianidis I, Flicek P, Odom DT: Five-vertebrate ChIP-seq reveals the evolutionary dynamics of transcription factor binding. Science 2010, 328:1036-1040.

23. CSAC: Initial sequence of the chimpanzee genome and comparison with the human genome. Nature 2005, 437:69-87.

24. Gibbs RA, Rogers J, Katze MG, Bumgarner R, Weinstock GM, Mardis ER, Remington KA, Strausberg RL, Venter JC, Wilson RK, Batzer MA, Bustamante CD, Eichler EE, Hahn MW, Hardison RC, Makova KD, Miller W, Milosavljevic A, Palermo RE, Siepel A, Sikela JM, Attaway T, Bell S, Bernard KE, Buhay CJ, Chandrabose MN, Dao M, Davis C, Delehaunty KD, Ding Y, et al: Evolutionary and biomedical insights from the rhesus macaque genome. Science 2007, 316:222-234.

25. Locke DP, Hillier LW, Warren WC, Worley KC, Nazareth LV, Muzny DM, Yang SP, Wang Z, Chinwalla AT, Minx P, Mitreva M, Cook L, Delehaunty KD, Fronick C, Schmidt H, Fulton LA, Fulton RS, Nelson JO, Magrini V, Pohl C, Graves TA, Markovic C, Cree A, Dinh HH, Hume J, Kovar CL, Fowler GR, Lunter G, Meader S, Heger A, et al: Comparative and demographic analysis of orang-utan genomes. Nature 2011, 469:529-533.

26. Scally A, Dutheil JY, Hillier LW, Jordan GE, Goodhead I, Herrero J, Hobolth A, Lappalainen T, Mailund T, Marques-Bonet T, McCarthy S, Montgomery SH, Schwalie PC, Tang YA, Ward MC, Xue Y, Yngvadottir B, Alkan C, Andersen LN, Ayub Q, Ball EV, Beal K, Bradley BJ, Chen Y, Clee CM, Fitzgerald S, Graves TA, Gu Y, Heath P, Heger A, et al: Insights into hominid evolution from the gorilla genome sequence. Nature 2012, 483:169-175.

27. Sugimoto $M$, Tahara $H$, Ide $T$, Furuichi $Y$ : Steps involved in immortalization and tumorigenesis in human B-lymphoblastoid cell lines transformed by Epstein-Barr virus. Cancer Res 2004, 64:3361-3364.

28. Barreiro LB, Marioni JC, Blekhman R, Stephens M, Gilad Y: Functional comparison of innate immune signaling pathways in primates. PLoS Genet 2010, 6:e1001249.

29. Blekhman R, Oshlack A, Chabot AE, Smyth GK, Gilad Y: Gene regulation in primates evolves under tissue-specific selection pressures. PLOS Genet 2008, 4:e1000271.

30. Brawand D, Soumillon M, Necsulea A, Julien P, Csardi G, Harrigan P, Weier M, Liechti A, Aximu-Petri A, Kircher M, Albert FW, Zeller U, Khaitovich P, Grutzner F, Bergmann S, Nielsen R, Paabo S, Kaessmann H: The evolution of gene expression levels in mammalian organs. Nature 2011, 478:343-348.

31. Khaitovich $P$, Enard W, Lachmann M, Paabo S: Evolution of primate gene expression. Nat Rev Genet 2006, 7:693-702.

32. Perry GH, Melsted P, Marioni JC, Wang Y, Bainer R, Pickrell JK, Michelini K, Zehr S, Yoder AD, Stephens M, Pritchard JK, Gilad Y: Comparative RNA sequencing reveals substantial genetic variation in endangered primates. Genome Res 2011, 22:602-610.

33. King MC, Wilson AC: Evolution at two levels in humans and chimpanzees. Science 1975, 188:107-116.

34. Cain CE, Blekhman R, Marioni JC, Gilad Y: Gene expression differences among primates are associated with changes in a histone epigenetic modification. Genetics 2011, 187:1225-1234.

35. Martin DI, Singer M, Dhahbi J, Mao G, Zhang L, Schroth GP, Pachter L, Boffelli D: Phyloepigenomic comparison of great apes reveals a correlation between somatic and germline methylation states. Genome Res 2011, 21:2049-2057.

36. Pai AA, Bell JT, Marioni JC, Pritchard JK, Gilad Y: A genome-wide study of DNA methylation patterns and gene expression levels in multiple human and chimpanzee tissues. PLoS Genet 2011, 7:e1001316.

37. Shibata Y, Sheffield NC, Fedrigo O, Babbitt CC, Wortham M, Tewari AK, London D, Song L, Lee BK, lyer VR, Partker SC, Margulies EH, Wray EH, Furey TS, Crawford GE: Extensive evolutionary changes in regulatory element activity during human origins are associated with altered gene expression and positive selection. PLOS Genet 2012, 8:e1002789.
38. Chabot A, Shrit RA, Blekhman R, Gilad Y: Using reporter gene assays to identify cis regulatory differences between humans and chimpanzees. Genetics 2007, 176:2069-2076.

39. Iskow RC, Gokcumen O, Abyzov A, Malukiewicz J, Zhu Q, Sukumar AT, Pai AA Mills RE, Habegger L, Cusanovich DA, Rubel MA, Perry GH, Gerstein M, Stone AC, Gilad Y, Lee C: Regulatory element copy number differences shape primate expression profiles. Proc Natl Acad Sci U S A 2012, 109:12656-12661.

40. Weth O, Renkawitz R: CTCF function is modulated by neighboring DNA binding factors. Biochem Cell Biol 2011, 89:459-468.

41. Zlatanova J, Caiafa P: CTCF and its protein partners: divide and rule? J Cell Sci 2009, 122:1275-1284.

42. Wang J, Lunyak W, Jordan IK: Genome-wide prediction and analysis of human chromatin boundary elements. Nucleic Acids Res 2011, 40:511-529.

43. Dunham I, Kundaje A, Aldred SF, Collins PJ, Davis CA, Doyle F, Epstein CB, Frietze S, Harrow J, Kaul R, Khatun J, Lajoie BR, Landt SG, Lee BK, Pauli F, Rosenbloom KR, Sabo P, Safi A, Sanyal A, Shoresh N, Simon JM, Song L, Trinklein ND, Altshuler RC, Birney E, Brown JB, Cheng C, Djebali S, Dong X, Dunham I, et al: An integrated encyclopedia of DNA elements in the human genome. Nature 2012, 489:57-74.

44. Guo Y, Mahony S, Gifford DK: High resolution genome wide binding event finding and motif discovery reveals transcription factor spatial binding constraints. PLoS Comput Biol 2012, 8:e1002638.

45. Shi Y, Seto E, Chang LS, Shenk T: Transcriptional repression by YY1, a human GLI-Kruppel-related protein, and relief of repression by adenovirus E1A protein. Cell 1991, 67:377-388.

46. Kim J, Kim JD: In vivo YY1 knockdown effects on genomic imprinting. Hum Mol Genet 2008, 17:391-401

47. Kim JD, Hinz AK, Bergmann A, Huang JM, Ovcharenko I, Stubbs L, Kim J: Identification of clustered $Y Y 1$ binding sites in imprinting control regions. Genome Res 2006, 16:901-911.

48. Kim JD, Hinz AK, Choo JH, Stubbs L, Kim J: YY1 as a controlling factor for the Peg3 and Gnas imprinted domains. Genomics 2007, 89:262-269.

49. Jeon $Y$, Lee JT: YY1 tethers Xist RNA to the inactive $X$ nucleation center. Cell 2011, 146:119-133.

50. Wu S, Hu YC, Liu H, Shi Y: Loss of YY1 impacts the heterochromatic state and meiotic double-strand breaks during mouse spermatogenesis. Mol Cell Biol 2009, 29:6245-6256.

51. Donohoe ME, Zhang X, McGinnis L, Biggers J, Li E, Shi Y: Targeted disruption of mouse Yin Yang 1 transcription factor results in peri-implantation lethality. Mol Cell Biol 1999, 19:7237-7244.

52. Atchison L, Ghias A, Wilkinson F, Bonini N, Atchison ML: Transcription factor YY1 functions as a PcG protein in vivo. EMBO J 2003, 22:1347-1358.

53. Brown JL, Mucci D, Whiteley M, Dirksen ML, Kassis JA: The Drosophila Polycomb group gene pleiohomeotic encodes a DNA binding protein with homology to the transcription factor YY1. Mol Cell 1998, 1:1057-1064.

54. Xi H, Yu Y, Fu Y, Foley J, Halees A, Weng Z: Analysis of overrepresented motifs in human core promoters reveals dual regulatory roles of YY1. Genome Res 2007, 17:798-806.

55. Schug J, Schuller WP, Kappen C, Salbaum JM, Bucan M, Stoeckert CJ Jr: Promoter features related to tissue specificity as measured by Shannon entropy. Genome Biol 2005, 6:R33.

56. Athanikar JN, Badge RM, Moran JV: A YY1-binding site is required for accurate human LINE-1 transcription initiation. Nucleic Acids Res 2004, 32:3846-3855.

57. Becker KG, Swergold GD, Ozato K, Thayer RE: Binding of the ubiquitous nuclear transcription factor $\mathrm{YY} 1$ to a cis regulatory sequence in the human LINE-1 transposable element. Hum Mol Genet 1993, 2:1697-1702.

58. Humphrey GW, Englander EW, Howard BH: Specific binding sites for a pol III transcriptional repressor and pol II transcription factor YY1 within the internucleosomal spacer region in primate Alu repetitive elements. Gene Expr 1996, 6:151-168

59. Knossl M, Lower R, Lower J: Expression of the human endogenous retrovirus HTDV/HERV-K is enhanced by cellular transcription factor YY1. J Virol 1999, 73:1254-1261.

60. Satyamoorthy K, Park K, Atchison ML, Howe CC: The intracisternal A-particle upstream element interacts with transcription factor $Y Y 1$ to activate transcription: pleiotropic effects of $Y Y 1$ on distinct DNA promoter elements. Mol Cell Biol 1993, 13:6621-6628.

61. Flicek P, Amode MR, Barrell D, Beal K, Brent S, Carvalho-Silva D, Clapham P Coates G, Fairley S, Fitzgerald S: Ensembl 2012. Nucleic Acids Res 2012, 40:D84-D90. 
62. Stefflova K, Thybert T, Wilson MD, Streeter I, Aleksic J, Karagianni P, Talianidis I, Brazma A, Adams D, Marioni J: Cooperativity and rapid evolution of co-bound transcription factors in closely related mammals. Cell 2013, 154:530-540.

63. Jacques $P E$, Jeyakani J, Bourque G: The majority of primate-specific regulatory sequences are derived from transposable elements. PLoS Genet 2013, 9:e1003504.

64. Liao D, Pavelitz T, Weiner AM: Characterization of a novel class of interspersed LTR elements in primate genomes: structure, genomic distribution, and evolution. J Mol Evol 1998, 46:649-660.

65. Cohen CJ, Lock WM, Mager DL: Endogenous retroviral LTRs as promoters for human genes: a critical assessment. Gene 2009, 448:105-114.

66. Ward MC, Wilson MD, Barbosa-Morais NL, Schmidt D, Stark R, Pan Q, Schwalie PC, Menon S, Lukk M, Watt S, Thybert D, Kutter C, Kirschner K, Flicek P, Blencowe BJ, Odom DT: Latent regulatory potential of human-specific repetitive elements. Mol Cell 2013, 49:262-272.

67. Xie X, Mikkelsen TS, Gnirke A, Lindblad-Toh K, Kellis M, Lander ES: Systematic discovery of regulatory motifs in conserved regions of the human genome, including thousands of CTCF insulator sites. Proc Natl Acad Sci U S A 2007, 104:7145-7150.

68. He Q, Bardet AF, Patton B, Purvis J, Johnston J, Paulson A, Gogol M, Stark A, Zeitlinger J: High conservation of transcription factor binding and evidence for combinatorial regulation across six Drosophila species. Nat Genet 2011, 43:414-420.

69. Tamura K, Subramanian S, Kumar S: Temporal patterns of fruit fly (Drosophila) evolution revealed by mutation clocks. Mol Biol Evol 2004 21:36-44.

70. Ni X, Zhang YE, Negre N, Chen S, Long M, White KP: Adaptive evolution and the birth of CTCF binding sites in the Drosophila genome. PLOS BiO 2012, 10:e1001420

71. Merkenschlager M, Odom DT: CTCF and cohesin: linking gene regulatory elements with their targets. Cell 2013, 152:1285-1297.

72. Bourque $G$, Leong $B$, Vega VB, Chen $X$, Lee $Y L$, Srinivasan $K G$, Chew JL, Ruan $Y$, Wei $\mathrm{CL}, \mathrm{Ng} \mathrm{HH}$, Liu ET: Evolution of the mammalian transcription factor binding repertoire via transposable elements. Genome Res 2008, 18:1752-1762.

73. Maksakova IA, Romanish MT, Gagnier L, Dunn CA, van de Lagemaat LN, Mager DL: Retroviral elements and their hosts: insertional mutagenesis in the mouse germ line. PLOS Genet 2006, 2:e2.

74. Waterston RH, Lindblad-Toh K, Birney E, Rogers J, Abril JF, Agarwal P, Agarwala R, Ainscough R, Alexandersson M, An P, Antonarakis SE, Attwood J, Baertsch R, Bailey J, Barlow K, Beck S, Berry E, Birren B, Bloom T, Bork P, Botcherby M, Bray N, Brent MR, Brown DG, Brown SD, Bult C, Burton J, Butler J, Campbell RD, Carninci P: Initial sequencing and comparative analysis of the mouse genome. Nature 2002, 420:520-562.

75. Kang K, Chung JH, Kim J: Evolutionary Conserved Motif Finder (ECMFinder) for genome-wide identification of clustered YY1- and CTCF-binding sites. Nucleic Acids Res 2009, 37:2003-2013.

76. Essien K, Vigneau S, Apreleva S, Singh LN, Bartolomei MS, Hannenhalli S: CTCF binding site classes exhibit distinct evolutionary, genomic, epigenomic and transcriptomic features. Genome Biol 2009, 10:R131.

77. Negre N, Brown CD, Ma L, Bristow CA, Miller SW, Wagner U, Kheradpour P, Eaton ML, Loriaux P, Sealfon R, Li Z, Ishii H, Spokony RF, Chen J, Hwang L, Cheng C, Auburn RP, Davis MB, Domanus M, Shah PK, Morrison CA, Zieba J, Suchy S, Senderowicz L, Victorsen A, Bild NA, Grundstad AJ, Hanley D, MacAlpine DM, Mannervik M: A cis-regulatory map of the Drosophila genome. Nature 2011, 471:527-531.

78. Rach EA, Winter DR, Benjamin AM, Corcoran DL, Ni T, Zhu J, Ohler U: Transcription initiation patterns indicate divergent strategies for gene regulation at the chromatin level. PLoS Genet 2011, 7:e1001274.

79. Chernukhin I, Shamsuddin S, Kang SY, Bergstrom R, Kwon YW, Yu W, Whitehead J, Mukhopadhyay R, Docquier F, Farrar D, Morrison I, Vigneron M, Wu SY, Chiang CM, Loukinov D, Lobanenkov V, Ohlsson R, Klenova E: CTCF interacts with and recruits the largest subunit of RNA polymerase II to CTCF target sites genome-wide. Mol Cell Biol 2007, 27:1631-1648.

80. Donohoe ME, Silva SS, Pinter SF, Xu N, Lee JT: The pluripotency factor Oct4 interacts with Ctcf and also controls X-chromosome pairing and counting. Nature 2009, 460:128-132.

81. Cai Y, Jin J, Yao T, Gottschalk AJ, Swanson SK, Wu S, Shi Y, Washburn MP, Florens L, Conaway RC, Conaway JW: YY1 functions with INO80 to activate transcription. Nat Struct Mol Biol 2007, 14:872-874.
82. Schmidt D, Wilson MD, Spyrou C, Brown GD, Hadfield J, Odom DT: ChIP-seq: using high-throughput sequencing to discover protein-DNA interactions. Methods 2009, 48:240-248.

83. Vella P, Barozzi I, Cuomo A, Bonaldi T, Pasini D: Yin Yang 1 extends the Myc-related transcription factors network in embryonic stem cells. Nucleic Acids Res 2012, 40:3403-3418.

84. Faure AJ, Schmidt D, Watt S, Schwalie PC, Wilson MD, Xu H, Ramsay RG, Odom DT, Flicek P: Cohesin regulates tissue-specific expression by stabilizing highly occupied cis-regulatory modules. Genome Res 2012, 22:2163-2175.

85. Langmead B: Aligning short sequencing reads with Bowtie. Curr Protoc Bioinformatics 2010, 11:Unit 11.7.

86. Xu H, Handoko L, Wei $X$, Ye C, Sheng J, Wei CL, Lin F, Sung WK: A signal-noise model for significance analysis of ChIP-seq with negative control. Bioinformatics 2010, 26:1199-1204.

87. Kent WJ, Sugnet CW, Furey TS, Roskin KM, Pringle TH, Zahler AM, Haussler D: The human genome browser at UCSC. Genome Res 2002, 12:996-1006.

88. Hedges SB, Dudley J, Kumar S: TimeTree: a public knowledge-base of divergence times among organisms. Bioinformatics 2006, 22:2971-2972.

89. Siepel A, Bejerano G, Pedersen JS, Hinrichs AS, Hou M, Rosenbloom K Clawson H, Spieth J, Hillier LW, Richards S, Weinstock GM, Wilson RK, Gibbs RA, Kent WJ, Miller W, Haussler D: Evolutionarily conserved elements in vertebrate, insect, worm, and yeast genomes. Genome Res 2005, 15:1034-1050.

90. Liu T, Ortiz JA, Taing L, Meyer CA, Lee B, Zhang Y, Shin H, Wong SS, Ma J, Lei Y, Pape UJ, Poidinger M, Chen Y, Yeung K, Brown M, Turpaz Y, Liu XS: Cistrome: an integrative platform for transcriptional regulation studies. Genome Biol 2011, 12:R83.

91. Kumar S, Subramanian S: Mutation rates in mammalian genomes. Proc Natl Acad Sci U S A 2002, 99:803-808.

92. Jurka J, Kapitonov W, Pavlicek A, Klonowski P, Kohany O, Walichiewicz J: Repbase Update, a database of eukaryotic repetitive elements. Cytogenet Genome Res 2005, 110:462-467.

93. Saldanha AJ: Java Treeview-extensible visualization of microarray data. Bioinformatics 2004, 20:3246-3248.

94. Down TA, Hubbard TJ: NestedMICA: sensitive inference of over-represented motifs in nucleic acid sequence. Nucleic Acids Res 2005, 33:1445-1453.

95. Bailey TL, Boden M, Buske FA, Frith M, Grant CE, Clementi L, Ren J, Li WW, Noble WS: MEME SUITE: tools for motif discovery and searching. Nucleic Acids Res 2009, 37:W202-W208.

96. Cerami EG, Bader GD, Gross BE, Sander C: cPath: open source software for collecting, storing, and querying biological pathways. BMC Bioinformatics 2006, 7:497.

97. McLean CY, Bristor D, Hiller M, Clarke SL, Schaar BT, Lowe CB, Wenger AM, Bejerano G: GREAT improves functional interpretation of cis-regulatory regions. Nat Biotechnol 2010, 28:495-501.

98. Turro E, Su SY, Gonçalves Â, Coin LJ, Richardson S, Lewin A: Haplotype and isoform specific expression estimation using multi-mapping RNA-seq reads. Genome Biol 2011, 12:R13.

doi:10.1186/gb-2013-14-12-r148

Cite this article as: Schwalie et al: Co-binding by YY1 identifies the transcriptionally active, highly conserved set of CTCF-bound regions in primate genomes. Genome Biology 2013 14:R148.

\section{Submit your next manuscript to BioMed Central and take full advantage of:}

- Convenient online submission

- Thorough peer review

- No space constraints or color figure charges

- Immediate publication on acceptance

- Inclusion in PubMed, CAS, Scopus and Google Scholar

- Research which is freely available for redistribution 